\title{
Comparison of Commercially Available Balanced Salt Solution and Ringer's Lactate on Extent of Correction of Metabolic Acidosis in Critically Ill Patients
}

\author{
Neha Rawat ${ }^{1}$, Neeru Sahni ${ }^{2}$, Lakshminarayana Yaddanapudi ${ }^{3}$
}

\begin{abstract}
Introduction: Appropriate early fluid resuscitation is ubiquitous for critically ill patients with metabolic acidosis. Owing to harmful effects of normal saline, commercially prepared balanced salt solutions are being used. However, there is no study comparing use of Ringer's lactate (RL) and commercially available balanced salt solutions in critically ill patients.

Materials and methods: A randomized controlled trial was conducted during July 2016 to December 2017. Fifty adult patients admitted to intensive care unit with metabolic acidosis were randomized into group RL or group acetate solution (AC). Respective trial fluid was administered at $20 \mathrm{~mL} / \mathrm{kg} /$ hour for first hour and $10 \mathrm{~mL} / \mathrm{kg} /$ hour for second hour. Arterial blood gas analysis samples were taken 15 minutes apart. The fluid resuscitation was continued till pH got corrected to 7.3 or 2 hours, whichever was earlier. The primary aim was to compare time to correct metabolic acidosis in both the groups. The secondary outcomes were the extent of correction of metabolic acidosis, total volume of fluid used, and total cost per patient.

Results: Demographic parameters, APACHE II score, and baseline investigations were comparable. The metabolic acidosis got corrected in 12 patients in group $\mathrm{AC}$ and 10 patients in group RL ( $p$ value $=0.66$ ). The mean time for correction of metabolic acidosis was $57 \pm 3.85$ minutes in group RL and $56.25 \pm 4.22$ minutes in group AC ( $p$ value $=0.95$ ). The extent of correction of metabolic acidosis and total volume of fluid used was also comparable ( $p$ value $=0.05$ ). However, the cost of fluid used was significantly higher in group AC $(p$ value $<0.01)$.

Conclusion: During administration of balanced salt solutions, RL or AC, in critically ill patients with metabolic acidosis, AC did not confer any advantage in time to or extent of correction of metabolic acidosis.

Clinical significance: There is no difference in acid-base status with use of different types of balanced salt solutions for resuscitation in critically ill patients.

Keywords: Acid base balance, Critically ill, Crystalloid solutions, Intensive care unit, Ringer's lactate.

Indian Journal of Critical Care Medicine (2020): 10.5005/jp-journals-10071-23488
\end{abstract}

\section{INTRODUCTION}

Metabolic acidosis in a critically ill patient is associated with worse outcome in terms of prolonged stay in intensive care unit (ICU). ${ }^{1}$ Appropriate early fluid resuscitation is recommended, as it increases cardiac output and organ perfusion, thereby improving the metabolic well-being which in turn decreases the base deficit. ${ }^{2}$ Owing to various harmful effects of normal saline (NS) ranging from hyperchloremic acidosis and endothelial glycocalyx damage to increased incidence of acute kidney injury and renal replacement therapy, balanced crystalloids containing physiologic levels of chloride and additional buffers are increasingly being used for critically ill patients. ${ }^{3}$ The literature for comparison of NS with balanced salt solutions in various clinical settings is abundant, but there is paucity of data when it comes to comparison of different balanced salt solutions. To the best of our knowledge, this is the first study with the primary aim of comparing the effects of two balanced salt solutions, widely available, Ringer's lactate $(R L)$ and commercially prepared, acetate solution (AC) on time to correct metabolic acidosis in critically ill patients admitted in ICU. The secondary outcomes assessed were the extent of correction of metabolic acidosis, total volume of fluid used, and the cost of two fluids.

\begin{abstract}
${ }^{1-3}$ Department of Anesthesia and Intensive Care, Postgraduate Institute of Medical Education and Research, Chandigarh, India

Corresponding Author: Neeru Sahni, Department of Anesthesia and Intensive Care, Postgraduate Institute of Medical Education and Research, Chandigarh, India, Phone: +91 9872646106, e-mail: neerunalin@yahoo.com

How to cite this article: Rawat N, Sahni N, Yaddanapudi L. Comparison of Commercially Available Balanced Salt Solution and Ringer's Lactate on Extent of Correction of Metabolic Acidosis in Critically III Patients. Indian J Crit Care Med 2020;24(7):539-543.
\end{abstract}

Source of support: PGIMER, Chandigarh

Conflict of interest: None

\section{Materials and Methods}

This was a single-center, randomized controlled trial (RCT) conducted from July 2016 to December 2017 in ICU of teaching tertiary-care center in North India. After obtaining institute's ethics committee approval (NK/2951/MD/1311), the trial was registered in clinical trials registry of India, CTRI/2017/08/009261. Adult patients admitted to the ICU with moderate or severe metabolic

() The Author(s). 2020 Open Access This article is distributed under the terms of the Creative Commons Attribution 4.0 International License (https://creativecommons. org/licenses/by-nc/4.0/), which permits unrestricted use, distribution, and non-commercial reproduction in any medium, provided you give appropriate credit to the original author(s) and the source, provide a link to the Creative Commons license, and indicate if changes were made. The Creative Commons Public Domain Dedication waiver (http://creativecommons.org/publicdomain/zero/1.0/) applies to the data made available in this article, unless otherwise stated. 
acidosis were randomized into two groups, group $\mathrm{RL}$ and group AC. Moderate metabolic acidosis was defined as pH 7.20 to 7.29 and standard bicarbonate 10 to $19 \mathrm{mmol} / \mathrm{L}$ and severe metabolic acidosis as $\mathrm{pH}<7.20$ with standard bicarbonate, $10 \mathrm{mmol} / \mathrm{L}^{4}$ Patient who did not give consent or had contraindication to volume resuscitation were excluded.

Assuming time taken for correction of metabolic acidosis as 60 minutes with administration of RL (initial 1 hour of fluid resuscitation) and a $20 \%$ decrease in this time (48 minutes) for AC, sample size was calculated as 11 in each group (total 22) with power of study at $80 \%$ and one-sided $p$ value as 0.05 to detect this difference. Since only about half patients will have correction of metabolic acidosis with fluid resuscitation alone, at least 22 patients were required in each group. To attribute for any loss of data, a total of 50 patients were enrolled and randomization was done by using a computer-generated random number chart for sequence generation, and sequentially numbered opaque-sealed envelopes were used for concealment of randomization. Once randomized further blinding of the intensivist collecting data were not possible due to obvious difference in packaging of two fluids. The statistical analysis was done by a person blinded to the group allocation.

After placing a $20-\mathrm{G}$ arterial canula in peripheral artery under all aseptic precautions, a baseline arterial blood gas (ABG) analysis was obtained: $S_{0}(0.5 \mathrm{~mL})$ for blood gas analysis, electrolytes, and lactate levels. After confirming metabolic acidosis in baseline ABG $\left(\mathrm{S}_{0}\right)$, fluid resuscitation with respective trial fluid was started at the rate of $20 \mathrm{~mL} / \mathrm{kg}$ for the first hour and $10 \mathrm{~mL} /$ hour for the second hour. Subsequent $A B G$ samples were drawn 15 minutes apart labeled $\mathrm{S}_{1}, \mathrm{~S}_{2}, \mathrm{~S}_{3}, \mathrm{~S}_{4}, \mathrm{~S}_{5}, \mathrm{~S}_{6}, \mathrm{~S}_{7}, \mathrm{~S}_{8}$ at 5, 30, 45, 60, 75, 90, 105, and 120 minutes, respectively, after initiation of resuscitation. Each patient continued to receive the same trial fluid till completion of the study.

The fluid resuscitation was continued till pH of 7.3 was achieved (considered as correction of metabolic acidosis) or for maximum of 2 hours, whichever was earlier. The extent of correction of metabolic acidosis was measured for patients in whom metabolic acidosis got corrected, in terms of change in $\mathrm{pH}$, standard bicarbonate, base excess, and lactate levels from beginning of fluid administration till time point at which metabolic acidosis got corrected. The total volume of fluid administered and the overall cost per patient were also recorded.

The statistical analysis was carried out using software $\mathrm{R}$ with package $\mathrm{R}$ commander. For all quantitative variables, mean and median were calculated. Standard deviation was calculated for measures of dispersion. Kolmogorov-Smirnov tests of normality were used to determine the distribution of data. Unpaired Student $t$ test was used for comparing normally distributed measurable data. Mann-Whitney $U$ test was used to compare skewed distributed data. These measurable data included age; height; weight; body mass index (BMI); baseline investigations such as hemogram, biochemistry (sodium, potassium, urea, creatinine, chloride and lactate); time to correction of acidosis; volume of IV fluid; cost of IV fluid; and APACHE score at 24 hours. Chi-square or Fisher exact test were used for categorical data. Repeated measure ANOVA test was used for time-related data to see the trend over time as well as compare the 2 groups at different points of time. These included correction of $\mathrm{pH}$, standard bicarbonate, base excess, and lactate.

\section{Results}

Fifty patients admitted to ICU during August 2016 to December 2017 meeting the inclusion criteria were recruited, 24 in group RL and 25 patients in group AC, and analyzed (Flowchart 1).
Demographic profile and baseline investigations of both the groups were comparable (Table 1). Median acute physiology and chronic health evaluation (APACHE) II score was 16 (14-20) in both the groups. The baseline acid-base status of patients in the two groups was comparable at the time of recruitment $\left(\mathrm{S}_{0}\right)$ (Table 2). The metabolic acidosis was corrected after fluid resuscitation in 12 (48\%) of the 25 patients in group AC, whereas the metabolic acidosis was corrected in 10 (41.6\%) of the 24 patients in group RL, and this difference was not statistically different ( $p$ value 0.66 ).

The primary outcome, that is, the mean time for correction of metabolic acidosis, was $57 \pm 3.85$ minutes in group $R L$ and $56.25 \pm$ 4.22 minutes in group $A C$, and the difference was not statistically significant ( $p$ value 0.95 ). The extent of correction of metabolic acidosis in patients who the metabolic acidosis got corrected was: Mean change in $\mathrm{pH}$ was $0.08 \pm 0.05$ in group $\mathrm{RL}$ and $0.11 \pm 0.09$ in group AC ( $p$ value 0.51 ), mean change in standard bicarbonate was $2.19 \pm 2.07 \mathrm{mEq} / \mathrm{L}$ in group $\mathrm{RL}$, and $2.46 \pm 2.26 \mathrm{mEq} / \mathrm{L}$ in group $\mathrm{AC}$ ( $p$ value 0.77 ), mean change in base excess was $2.73 \pm 2.21 \mathrm{mEq} / \mathrm{L}$ in group $\mathrm{RL}$ and $3.28 \pm 3.25 \mathrm{mEq} / \mathrm{L}$ in group $\mathrm{AC}$ ( $p$ value 0.64 ,) and mean fall in lactate was $1.31 \pm 3.56 \mathrm{mmol} / \mathrm{L}$ in group $\mathrm{RL}$ and $0.03 \pm$ $1.79 \mathrm{mmol} / \mathrm{L}$ in group AC ( $p$ value $=0.32$ ) (Figs 1 and 2 ).

The mean volume of total IV fluid used for resuscitation was $1513.75 \pm 511.13 \mathrm{~mL}$ in group RL and $1472.52 \pm 521.13 \mathrm{~mL}$ in group AC ( $p$ value $=0.78$ ). However, the mean total cost of IV fluid (in Indian rupee) was $172.9 \pm 53.6$ in group $\mathrm{RL}$ and $336 \pm 111.4$ in group AC ( $p$ value $<0.01)$.

\section{Discussion}

The study showed that no particular balanced salt solution (RL or AC) conferred advantage over the other in terms of correction of metabolic acidosis and extent to which the correction happened. The number of patients in who the metabolic acidosis got corrected with initial fluid resuscitation for 2 hours remained the same in both the groups. However, the use of commercially prepared balanced crystalloid, AC, did increase the overall cost of treatment when compared to widely available cheaper balanced solution, RL.

The commercially prepared balanced salt solutions come with different buffers and are promoted with data from clinical studies emphasizing their superiority over NS. ${ }^{5-9}$ Interestingly, the number of studies comparing the commercially prepared costlier solutions with $\mathrm{RL}$ is limited. The choice of fluid to be administered in resuscitation of critically ill patients in an ICU is a matter of utmost significance. The availability and cost of fluid being recommended for administration are equally significant when aiming to achieve the 1-hour bundle goal per Surviving Sepsis Guidelines. ${ }^{10}$ Hence, the comparison of RL and AC is logical because RL is cheaper and widely available balanced salt solution.

Among the three studies comparing balanced salt solutions, Hadimioglu and colleagues conducted a randomized controlled trial and compared administration of AC, NS, and RL in renal transplant recipients. ${ }^{11}$ They concluded that the acid-base parameters were similar to both balanced fluids, RL and AC. However, lactate levels were significantly higher in patients receiving $\mathrm{RL}$ intraoperatively which is in contrast to our study, where no difference in lactate levels was found. The reason could be the different patient population and difference in volume of fluid administered in the two studies.

Weinberg et al. studied the effect of peri-operatively administering two balanced salt solutions, Hartmann solution (HS) and plasmalyte (PL), in patients undergoing major hepatic resection and concluded that patients in both the groups had equivalent 
Flowchart 1: CONSORT study flowchart

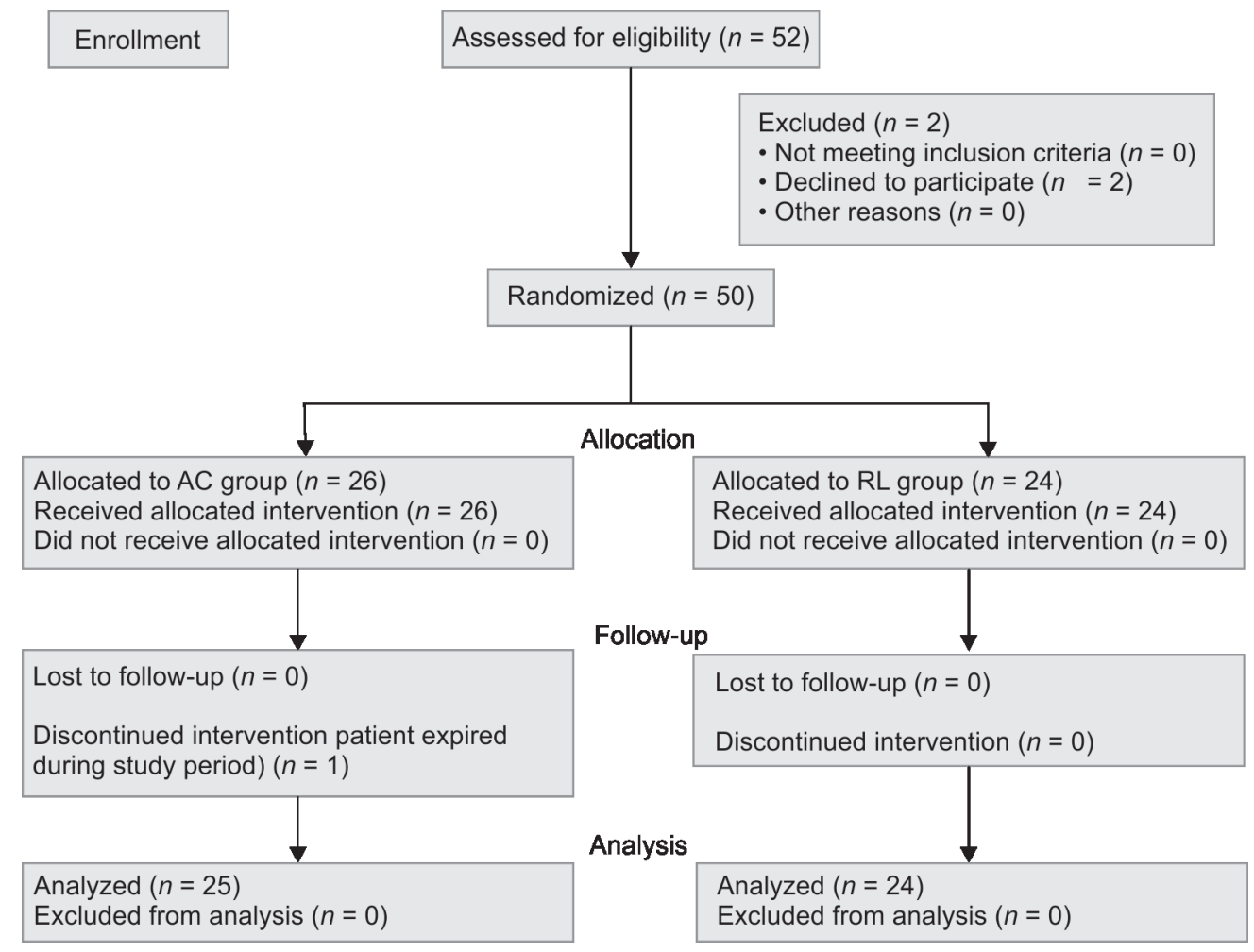

Table 1: Demographic parameters and baseline investigations

\begin{tabular}{lll}
\hline Parameter & Group RL & Group AC \\
\hline Age (years) & $42.9 \pm 18.9$ & $48.6 \pm 18.0$ \\
$\begin{array}{l}\text { Gender: male/female } \\
\text { (numbers) }\end{array}$ & $15 / 9$ & $15 / 10$ \\
Weight (kg) & $62.9 \pm 9.2$ & $61.2 \pm 8.0$ \\
Height (meter) & $1.6 \pm 0.1$ & $1.6 \pm 0.1$ \\
Hemoglobin (g/dL) & $9.80 \pm 1.8$ & $9.9 \pm 2.1$ \\
Platelet count (per dL) & $136,000 \pm 89,109.1$ & $116,840 \pm 64397.6$ \\
Leukocyte (per dL) & $16395.8 \pm 9488.5$ & $19828.0 \pm 15084.2$ \\
Sodium (mEq/L) & $142.5 \pm 6.9$ & $138.22 \pm 6.4$ \\
Potassium (mEq/L) & $3.9 \pm 0.7$ & $4.2 \pm 0.91$ \\
Blood urea (mg/dL) & $63.9 \pm 23.7$ & $69.3 \pm 38.6$ \\
Serum creatinine (mg/dL) & $1.8 \pm 1.9$ & $1.8 \pm 1.1$ \\
\hline
\end{tabular}

*All values expressed in mean \pm standard deviation

mean standard base excess ( $p$ value 0.17$).{ }^{12}$ However, there was significant difference in serum chloride and serum lactate levels with patients in HS group being hyperchloremic ( $p$ value 0.03 ) and hyperlactatemic ( $p$ value 0.01 ).

Also, Shin et al. compared the effects of administering RL and plasmalyte in donors of liver transplant, undergoing right hepatectomy, and found higher lactate level, total peak bilirubin, and prothrombin times in patients receiving RL. ${ }^{13}$ Thus, the authors concluded that using balanced salt solutions without lactate as buffer is advantageous in donors of liver transplantation. The reason for the difference between this study and our study can be explained by the fact that despite critical illness, their liver function was well preserved in our patients, and lactate from RL was effectively metabolized.

Table 2: Parameters of arterial blood gas analysis (acid-base status) at the time of recruitment $\left(\mathrm{S}_{0}\right)$

\begin{tabular}{lccl}
\hline Parameter & Group $R L$ & Group $A C$ & $p$ value \\
\hline $\mathrm{pH}$ & $7.18 \pm 0.07$ & $7.19 \pm 0.08$ & 0.63 \\
$\mathrm{pO}_{2}(\mathrm{~mm} \mathrm{Hg})$ & $119.6 \pm 90.2$ & $152.6 \pm 118$ & 0.28 \\
$\mathrm{pCO}_{2}(\mathrm{~mm} \mathrm{Hg})$ & $40.9 \pm 11.8$ & $36.3 \pm 10.4$ & 0.15 \\
Standard bicarbonate & $14.9 \pm 2.9$ & $14.1 \pm 3.3$ & 0.91 \\
(mEq/L) & & & \\
Base deficit $(\mathrm{mEq} / \mathrm{L})$ & $-12.3 \pm 3.8$ & $-12.9 \pm 4.2$ & 0.68 \\
Chloride $(\mathrm{mEq} / \mathrm{L})$ & $116.8 \pm 10.5$ & $116.9 \pm 7.6$ & 0.97 \\
Lactate $(\mathrm{mMol} / \mathrm{L})$ & $4.0 \pm 3.7$ & $3.5 \pm 3.9$ & 0.5 \\
\hline
\end{tabular}

${ }^{*} p$ value $<0.05$ significant

Apart from these studies comparing different balanced salt solutions in different clinical settings, there are studies in which authors have used different crystalloids for patients with metabolic acidosis. Chua et al. published a retrospective analysis of almost exclusive resuscitation with AC and NS in patients with diabetic ketoacidosis and concluded faster correction, higher serum bicarbonate level and base excess with the use of AC ( $p$ value $<$ $0.05) .{ }^{14}$ The total volume of AC used was also significantly lower ( $p$ value $<0.001)$. Mahler et al., also compared AC with NS in diabetic ketoacidosis and confirmed lower bicarbonate levels ( $p$ value $<$ 0.05 ) and higher chloride levels with the use of NS ( $p$ value $<0.01) .^{15}$ In trauma patients with metabolic acidosis, Young et al. compared resuscitation for first 24 hours with AC and NS. ${ }^{9}$ Patients receiving $A C$ had larger change in serum bicarbonate, level base excess, and lower hyperkalemia at 24 hours postinjury. The major drawback of all these studies conducted in patients with metabolic acidosis is that they have compared NS with AC. Despite availability of a 

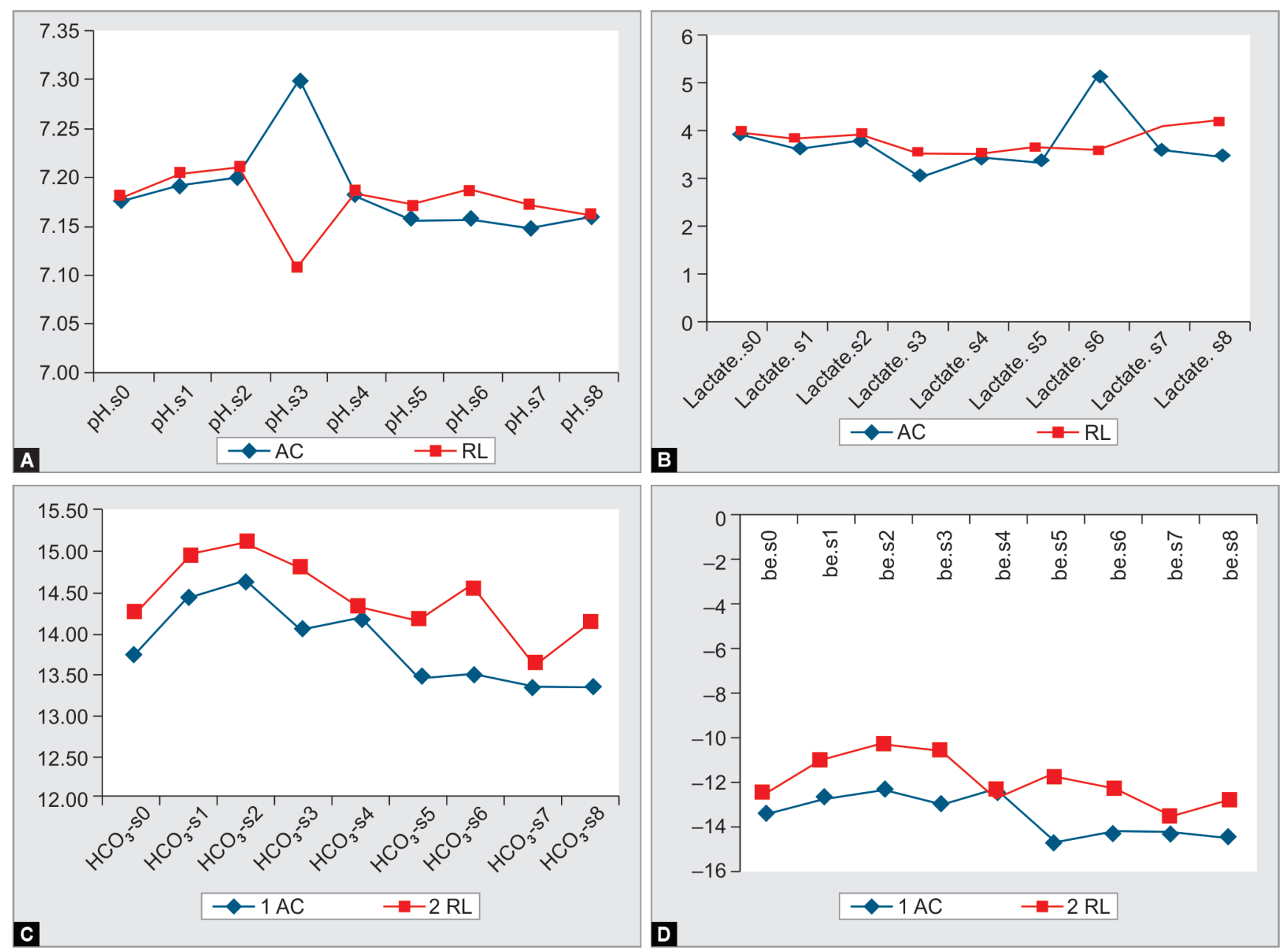

Figs $1 \mathrm{~A}$ to D: Extent of correction of metabolic acidosis in both groups in terms of: (A) pH; (B); Lactate level; (C) Bicarbonate ( $\left.\mathrm{HCO}_{3}{ }^{-}\right)$; (D) Base excess

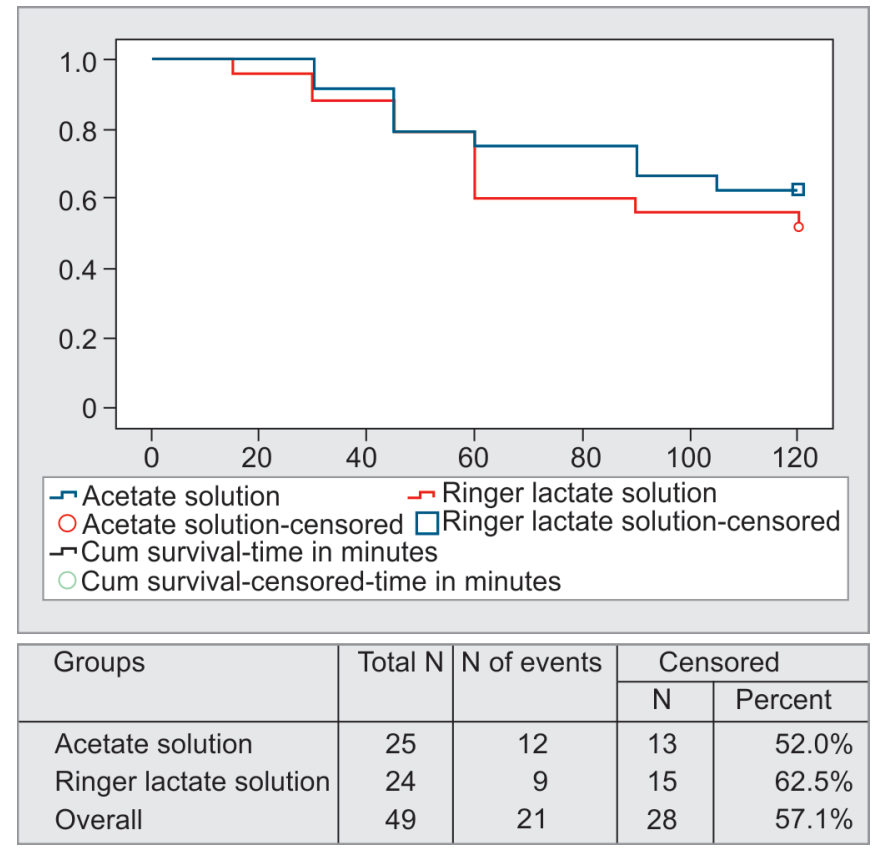

Fig. 2: Kaplan-Meier graph showing the number of patients with correction of metabolic acidosis with time in the two groups cheaper solution, $\mathrm{RL}$, there is no study comparing $\mathrm{RL}$ and $\mathrm{AC}$ in patients with metabolic acidosis.

In an animal model of hemorrhagic shock, Noritomi et al. studied AC, RL, and NS. ${ }^{16}$ Comparable $\mathrm{pH}$, serum bicarbonate, base excess, and chloride levels were found when study parameters in two groups AC and RL were analyzed. However, when NS was compared to balanced fluids, there was significant difference in serum bicarbonate ( $p$ value 0.04 ), base excess ( $p$ value 0.04 ), and chloride levels ( $p$ value 0.02).

Our study has certain limitations. First, our ICU is a medical ICU, and diagnosis at admission for majority of patients is sepsis. The extrapolation of results may not be feasible in ICU's admitting a different patient profile (surgical, trauma, and burn). Second, the study period was limited to 2 hours, considering the initial phase of fluid resuscitation in sepsis patients. We did not study the long-term outcomes such as incidence of AKI, need for RRT, mean duration of ICU stay, mean days of mechanical ventilation, and mortality.

\section{Conclusion}

During administration of 2 balanced salt solutions, RL and commercially prepared AC, to critically ill patients with metabolic acidosis during initial phase of fluid resuscitation, AC did not confer any advantage in time to or extent of correction of metabolic 
acidosis and the total volume of fluid used. However, use of AC increased the total cost as compared to RL.

\section{Clinical Significance}

There is no difference in acid-base balance with use of different types of balanced salt solutions for resuscitation in critically ill patients.

\section{Presented in}

31st Annual Congress of European Society of Intensive Care Medicine LIVES 2018 at Paris, France from October 20-24th, 2018.

\section{Abstract Published in}

ESICM LIVES 2018: Paris, France. 20-24 October 2018. Intensive Care Med Exp. 2018;6(Suppl 2):40. Published 2018 Oct 19. DOI: 10.1186/ s40635-018-0201-6

\section{References}

1. Kellum JA. Metabolic acidosis in patients with sepsis: epiphenomenon or part of the pathophysiology? Crit Care Resusc 2004;6(3):197-203.

2. Skellett S, Mayer A, Durward A, Tibby SM, Murdoch IA. Chasing the base deficit: hyperchloraemic acidosis following $0.9 \%$ saline fluid resuscitation. Arch Dis Child 2000;83(6):514-516. DOI: 10.1136/ adc.83.6.514.

3. Myburgh JA, Mythen MG. Resuscitation fluids. N Engl J Med 2013;369(13):1243-1251. DOI: 10.1056/NEJMra1208627.

4. Kraut JA, Madias NE. Treatment of acute metabolic acidosis: a pathophysiologic approach. Nat Rev Nephrol 2012;8(10):589-601. DOI: 10.1038/nrneph.2012.186.

5. Semler MW, Self WH, Wanderer JP, Ehrenfeld JM, Wang L, et al. Balanced crystalloid versus saline in critically ill adults. N Engl J Med 2018;378(9):829-839. DOI: 10.1056/NEJMoa1711584.

6. Liu C, Mao Z, Hu P, Hu X, Kang H, Hu J, et al. Fluid resuscitation in critically ill patients: a systematic review and network meta-analysis. Ther Clin Risk Manag 2018;14:1701-1709. DOI: 10.2147/TCRM.S175080.
7. Chowdhury AH, Cox EF, Francis ST, Lobo DN. A randomized, controlled, double-blind crossover study on the effects of $2-\mathrm{L}$ infusions of $0.9 \%$ saline and plasmalyte (R) 148 on renal blood flow velocity and renal cortical tissue perfusion in healthy volunteers. Ann Surg 2012;256(1):18-24. DOI: 10.1097/SLA.0b013e318256be72.

8. Shaw AD, Bagshaw SM, Goldstein SL, Scherer LA, Duan M, Schermer $\mathrm{CR}$, et al. Major complications, mortality and resource utilization after open abdominal surgery: $0.9 \%$ saline compared to Plasma-Lyte. Ann Surg 2012;255(5):821-829. DOI: 10.1097/SLA.0b013e31825074f5.

9. Young JB, Utter GH, Schermer CR, Galante JM, Phan HH, Yang Y, et al. Saline versus plasmalyte a in initial resuscitation of trauma patients: a randomized trial. Ann Surg 2014;259(2):255-262. DOI: 10.1097/ SLA.0b013e318295feba.

10. Casey JD, Brown RM, Semler MW. Resuscitation fluids. Curr Opin Crit Care 2018;24(6):512-518. DOI: 10.1097/MCC.0000000000000551.

11. Hadimioglu N, Saadawy I, Saglam T, Ertug Z, Dinckan A. The effect of different crystalloid solutions on acid-base balance and early kidney function after kidney transplantation. Anesth Analg 2008;107(1):264269. DOI: 10.1213/ane.0b013e3181732d64.

12. Weinberg L, Pearce B, Sullivan R, Siu L, Scurrah N, Tan C, et al. The effects of plasmalyte-148 vs Hartmann's solution during major liver resection: a multicenter, double-blind, randomized controlled trial. Minerva Anesthesiol 2015;81(12):1288-1297.

13. Shin WJ, Kim YK, Bang JY, Cho SK, Han SM, Hwang GS. Lactate and liver function tests after living donor right hepatectomy: a comparison of solutions with and without lactate. Acta Anaesthesiol Scand 2011;55(5):558. DOI: 10.1111/j.1399-6576.2011.02398.x-64.

14. Chua HR, Venkatesh B, Stachowski E, Schneider AG, Perkins K, Ladanyi $S$, et al. Plama-lyte 148 vs $0.9 \%$ saline for fluid resuscitation in diabetic ketoacidosis. J Crit Care 2012;27(2):138-145. DOI: 10.1016/j. jcrc.2012.01.007.

15. Mahler SA, Conrad SA, Wang H, Arnold TC. Resuscitation with balanced electrolyte solution prevents hyperchloremic metabolic acidosis in patients with diabetic ketoacidosis. Am J Emerg Med 2011;29(6):670-674. DOI: 10.1016/j.ajem.2010.02.004.

16. Noritomi DT, Pereira AJ, Bugano DD, Rehder PS, Silva E. Impact of plasmalyte $\mathrm{pH}-7.4$ on acid base status and haemodynamics in a model of controlled hemorrhagic shock. Clinics (Sao Paulo) 2011;66(11):1969-1974. DOI: 10.1590/S1807-59322011001100019. 